\title{
SECOND QUANTIZED AUTOMORPHISMS OF THE RENORMALIZED SQUARE OF WHITE NOISE (RSWN) ALGEBRA
}

\author{
LUIGI ACCARDI*, GRIGORI AMOSOV and UWE FRANZ \\ Centro Vito Volterra, Facaltá di Economia, \\ Università di Roma Tor Vergata, Via di Tor Vergata, \\ 00133 Roma, Italy \\ *accardi@volterra.mat.unirvmal.it
}

Received 10 November 2002

Revised 18 November 2003

Communicated by Y. G. Lu

\begin{abstract}
We determine the structure of the *-endomorphisms of the RSWN algebra, induced by linear maps in the 1-particle Hilbert algebra, introduce the RSWN analogue of the free evolutions and find the explicit form of the KMS states associated with some of them.
\end{abstract}

\section{The Renormalized Square of White Noise and Its Representations}

The renormalized square of white noise (RSWN) and its Fock representation were introduced in Ref. 1. It was shown in Ref. 2 that one can associate to each irreducible unitary representations of $\mathbf{s l}_{2}$ a number of representations of the renormalized square of white noise (RSWN) algebra $\mathcal{A}$. However, in analogy with the boson, fermion and $q$-deformed case, we expect that some interesting non-irreducible representations of $\mathbf{s l}_{2}$ may arise from the GNS representations of KMS states associated with some evolutions on $\mathcal{A}$. In the case of first-order fields the simplest evolutions are obtained as second quantizations of 1-particle evolutions, i.e. 1-parameter unitary groups on the test function space.

In this case in fact any unitary operator on the 1-particle space induces a $*$ automorphism of the $*$-Lie algebra defined by the first-order commutation relations (in unbounded form)

$$
\left[A_{f}, A_{g}^{+}\right]=\langle f, g\rangle \text {. }
$$

Such automorphisms are called free (or quasi-free). Conversely, if an automorphism $u$ of the Lie algebra generated by the $A_{f}^{+}, A_{f}(f \in \mathcal{H})$ and 1 is the second quantization of a map $V: \mathcal{H} \rightarrow \mathcal{H}$, then

$$
\left[u\left(A_{f}\right), u\left(A_{g}^{+}\right)\right]=\left[A_{V f}, A_{V g}^{+}\right]=\langle V f, V g\rangle=u\left(\left[A_{f}, A_{g}^{+}\right]\right)=u(\langle f, g\rangle)=\langle f, g\rangle,
$$


i.e. $V$ must be unitary. Our first goal is to solve the analogous problem for the RSWN algebra. Let us remark, incidentally, that the symmetry group of a set of commutation relations depends on the choice of the generators of the Lie algebra.

This important point will be discussed elsewhere.

In Sec. 2 we introduce the quasifree automoprhisms of $\mathcal{A}$, we obtain a classification of these automorphisms and prove their spatiality. Once this is done, we have a natural family of "free evolutions" on this algebra and we can investigate the structure of the KMS states with respect to these free evolutions. We begin to realize this program in Sec. 3 where we consider the KMS states associated with a special subclass of the quasifree evolutions on $\mathcal{A}$.

Recently Franz ${ }^{3}$ has shown that our construction of the KMS state generalizes to inner automorphisms of general bialgebras, if they are implemented by a trace class element.

In the remaining of this section we recall some definitions, and properties of the RSWN algebra.

For simplicity of notation we only discuss the RSWN over $\mathbb{R}$, but all the arguments below apply, without change, to the case of $\mathbb{R}^{d}$ with $d \geq 1$, i.e. for general quantum fields.

Definition 1. The square of white noise (SWN) algebra $\mathcal{A}$ over the Hilbert algebra $\mathcal{K}=L^{2}(\mathbf{R}) \cap L^{\infty}(\mathbf{R})$ (see Refs. 1 and 2 ), is the unital $*$-Lie algebra with generators 1 (central element), $b_{\phi}, b_{\phi}^{+}, n_{\phi}, \phi \in \mathcal{K}$, which are linearly independent (in the sense that $f_{0} 1+b_{f_{1}}+b_{f_{2}}+n_{f_{3}}=0$ with $f_{0} \in \mathbf{C}$ and $f_{j} \in \mathcal{K}(j=1,2,3)$ if and only if $f_{0}=f_{1}=f_{2}=f_{3}=0$ ) and relations

$$
\begin{aligned}
{\left[b_{\phi}, b_{\psi}^{+}\right] } & =\gamma\langle\phi, \psi\rangle 1+n_{\bar{\phi} \psi}, \\
{\left[n_{\phi}, b_{\psi}\right] } & =-2 b_{\bar{\phi} \psi}, \\
{\left[n_{\phi}, b_{\psi}^{+}\right] } & =2 b_{\phi \psi}^{+}, \\
\left(b_{\phi}\right)^{*} & =b_{\phi}^{+}, \quad\left(n_{\phi}\right)^{*}=n_{\bar{\phi}}, \\
{\left[n_{\phi}, n_{\psi}\right] } & =\left[b_{\phi}^{+}, b_{\psi}^{+}\right]=\left[b_{\phi}, b_{\psi}\right]=0
\end{aligned}
$$

where $\gamma$ is a fixed strictly positive real parameter (coming from the renormalization), $\langle\cdot, \cdot\rangle$ is an inner product in $\mathcal{K}$ and $\phi, \psi \in \mathcal{K}$. Furthermore $b^{+}$and $n$ are linear and $b$ is anti-linear in the functions from $\mathcal{K}$ and we assume that

$$
X_{\phi}=0 \text { if and only if } \phi=0
$$


Remark 1. The involution on $\mathcal{K}$ will be indifferently denoted $f \mapsto f^{*}$ or $f \mapsto \bar{f}$. It is convenient to think of the elements of $\mathcal{K}$ as multiplication operators on $L^{2}(\mathbb{R})$. In particular, $f=0$ means $f=0$ a.e. for the Lebesgue measure.

Following Ref. 2 a class of unitary representations of $\mathcal{A}$ can be obtained as follows. Consider the Lie algebra $\mathbf{s l}_{2}$ with generators $\left\{B^{+}, B^{-}, M\right\}$, relations

$$
\left[B^{-}, B^{+}\right]=M,\left[M, B^{ \pm}\right]= \pm B^{ \pm},
$$

and involution $\left(B^{-}\right)^{*}=B^{+}, M^{*}=M$. Let $\rho$ be an irreducible representation of $\mathbf{s l}_{2}$ and therefore of its universal enveloping unital algebra $\mathcal{U}\left(\mathbf{s l}_{2}\right)$ acting on a Hilbert space $\mathcal{H}_{0}$ and let $\eta$ be a 1-cocycle for $\rho$. Define $L(u v)=\left\langle\eta\left(u^{*}\right), \eta(v)\right\rangle, u$, $v \in \mathcal{U}_{0}\left(\mathbf{s l}_{2}\right)$, where $\mathcal{U}_{0}\left(\mathbf{s l}_{2}\right)$ is the algebra $\mathcal{U}\left(\mathbf{s l}_{2}\right)$ without unit. Then $(\rho, \eta, L)$ is called a Schürmann triple and a representation $\pi$ of $\mathcal{A}$ in the Hilbert space $\Gamma\left(\mathcal{H}_{0} \otimes L^{2}(\mathbf{R})\right)$ can be obtained from the relations

$$
\begin{aligned}
& \pi\left(b_{\chi_{[s, t[}}\right)=\Lambda_{s t}\left(\rho\left(B^{-}\right)\right)+A_{s t}^{*}\left(\eta\left(B^{-}\right)\right)+A_{s t}\left(\eta\left(B^{+}\right)\right)+L\left(B^{-}\right)(t-s) \mathrm{Id}, \\
& \pi\left(b_{\chi_{[s, t[}}^{+}\right)=\Lambda_{s t}\left(\rho\left(B^{+}\right)\right)+A_{s t}^{*}\left(\eta\left(B^{+}\right)\right)+A_{s t}\left(\eta\left(B^{-}\right)\right)+L\left(B^{+}\right)(t-s) \mathrm{Id}, \\
& \pi\left(n_{\chi_{[s, t[}}\right)=\Lambda_{s t}(\rho(M))+A_{s t}^{*}(\eta(M))+A_{s t}(\eta(M))+(L(M)-\gamma)(t-s) \mathrm{Id},
\end{aligned}
$$

where $\chi_{I}(I \subset \mathbf{R})$ denotes the multiplication operator by the characteristic function of $I$ ( $=1$ on $I$ and $=0$ outside $I), \gamma$ is the same as in $\left(\mathrm{SWN}_{1}\right) . \Lambda_{s t}=\Lambda_{t}-\Lambda_{s}$, $A_{s t}^{*}=A_{t}^{*}-A_{s}^{*}, A_{s t}=A_{t}-A_{s}$ are the conservation, creation and annihilation processes on the symmetric Fock space $\Gamma\left(\mathcal{H}_{0} \otimes L^{2}(\mathbf{R})\right)$ satisfying the relations (see Ref. 4) and

$$
\begin{gathered}
{\left[A_{t}(\phi), A_{s}^{*}(\psi)\right]=(t \wedge s)\langle\phi, \psi\rangle,} \\
{\left[A_{t}(\phi), A_{s}(\psi)\right]=\left[A_{t}^{*}(\phi), A_{s}^{*}(\psi)\right]=0,} \\
{\left[\Lambda_{t}(X), \Lambda_{s}(Y)\right]=\Lambda_{t \wedge s}([X, Y]),} \\
{\left[\Lambda_{t}(X), A_{s}(\phi)\right]=-A_{t \wedge s}\left(X^{*} \phi\right),\left[\Lambda_{t}(X), A_{s}^{*}(\phi)\right]=A_{t \wedge s}^{*}(X \phi) .}
\end{gathered}
$$

Here $\phi, \psi \in \mathcal{K}$ and $X, Y \in \mathcal{B}(\mathcal{K})$, i.e. they are linear operators on $L^{2}(\mathbb{R})$ leaving the subspace $\mathcal{K}$ invariant.

Conversely, all the irreducible representations of $\mathcal{A}$ arise in this way. We shall say that the representation $\rho$ is associated with the representation $\pi$ and the cocycle $\eta$. Hence to classify the representations of $\mathcal{A}$ one needs to investigate the representations of $\mathcal{U}\left(\mathbf{s l}_{2}\right)$ and their cocycles.

\section{Endomorphisms of the SWN Algebra}

Definition 2. A Hilbert algebra is a $*$-algebra $\mathcal{K}$, not necesarily with unit, endowed with a scalar product $\langle f, g\rangle \in \mathbf{C}$ satisfying $\langle f, g h\rangle=\left\langle g^{*} f, h\right\rangle(f, g, h \in \mathcal{K})$. A Hilbert 
algebra endomorphism (resp. automorphism) of $\mathcal{K}$ is a $*$-endomorphism (resp. *automorphism) $T$ of the $*$-algebra structure

$$
T(\phi \psi)=T(\phi) T(\psi), \quad(T(\phi))^{*}=T(\bar{\phi})
$$

which is also an isometry (resp. unitary operator), of the pre-Hilbert space structure in the sense that, $\forall \phi, \psi \in \mathcal{K}$

$$
\langle T(\phi), T(\psi)\rangle=\langle\phi, \psi\rangle \text {. }
$$

Theorem 1. Let $T^{1}, T^{2}, T^{3}$ be linear operators on $\mathcal{K}$. Define a map $\tau^{\prime}$ acting on the generators $b, b^{+}, n$ by the formula

$$
1 \rightarrow 1 ; \quad b_{\phi} \rightarrow b_{T^{1} \phi}, \quad b_{\phi}^{+} \rightarrow b_{T^{2} \phi}^{+}, \quad n_{\phi} \rightarrow n_{T^{3} \phi} .
$$

The map $\tau^{\prime}$ can be extended to a *-endomorphism of $\mathcal{A}$ if and only if there exist a Hilbert algebra endomorphism $T$ of $\mathcal{K}$ and a real-valued function $\alpha$ on $\mathbf{R}$, such that, for any $\phi \in \mathcal{K}$,

$$
\begin{aligned}
& T^{3}(\phi)=T(\phi), \\
& T^{1}(\phi)=T^{2}(\phi)=e^{i \alpha} T(\phi) .
\end{aligned}
$$

The endomorphism $\tau^{\prime}$ is an automorphism if and only if $T$ is an automorphism.

Proof. Due to the linear independence of the generators and to $\left(\mathrm{SWN}_{6}\right)$, it follows from the relation $\left(\mathrm{SWN}_{1}\right)$ that $\tau^{\prime}$, defined by $(2)$, is a Lie algebra automorphism if and only if

$$
\left(T^{1}\right)^{*} T^{2}=\mathrm{Id}, \quad T^{3}(\bar{\phi} \psi)=\overline{T^{1}(\phi)} T^{2}(\psi),
$$

where the first identity in condition (5) has to be interpreted in the sense of (1). From $\left(\mathrm{SWN}_{2}\right)$ and $\left(\mathrm{SWN}_{6}\right)$ it follows that

$$
T^{1}(\bar{\phi} \psi)=\overline{T^{3}(\phi)} T^{1}(\psi),
$$

from $\left(\mathrm{SWN}_{3}\right)$ and $\left(\mathrm{SWN}_{6}\right)$, that

$$
T^{2}(\phi \psi)=T^{3}(\phi) T^{2}(\psi)
$$

and from $\left(\mathrm{SWN}_{4}\right)$ and $\left(\mathrm{SWN}_{6}\right)$, that

$$
T_{0}:=T^{1}=T^{2}, \quad \overline{T^{3}(\phi)}=T^{3}(\bar{\phi}),
$$

for any $\phi, \psi \in \mathcal{K}$. Therefore (5)-(8) are respectively equivalent to:

$$
\begin{aligned}
T_{0}^{*} T_{0} & =\mathrm{Id}, \\
T^{3}(\bar{\phi}) & =\overline{T^{3}(\phi)}, \\
T^{3}(\bar{\phi} \psi) & =\overline{T_{0}(\phi)} T_{0}(\psi), \\
T_{0}(\phi \psi) & =T^{3}(\phi) T_{0}(\psi) .
\end{aligned}
$$


From (11) with $\phi=\psi$, we deduce that for any $\psi \in \mathcal{K}$

$$
T^{3}\left(|\psi|^{2}\right)=\left|T_{0}(\psi)\right|^{2} .
$$

Again from (11), with $\psi$ replaced by $\psi \chi$ we obtain

$$
T^{3}(\bar{\phi} \psi \chi)=\overline{T_{0}(\phi)} T_{0}(\psi \chi)
$$

and, from (12) this is equal to

$$
\overline{T_{0}(\phi)} T^{3}(\psi) T_{0}(\chi) .
$$

Choosing $\phi=\chi$ and using (13) we deduce

$$
T^{3}\left(|\phi|^{2} \psi\right)=\left|T_{0}(\phi)\right|^{2} T^{3}(\psi)=T^{3}\left(|\phi|^{2}\right) T^{3}(\psi) .
$$

Since a dense set of positive elements in $\mathcal{K}$ can be written in the form $|\phi|^{2}$ for some $\phi \in \mathcal{K}$, this implies that, for dense set of positive elements $\phi$ in $\mathcal{K}$

$$
T^{3}(\phi \psi)=T^{3}(\phi) T^{3}(\psi) .
$$

Since any $\phi \in \mathcal{K}$ is a linear combination of positive elements we conclude that (14) holds for any $\phi, \psi \in \mathcal{K}$.

Combining (13) and (14) we conclude that, for any $\psi \in \mathcal{K}$

$$
\left|T^{3}(\psi)\right|^{2}=\left|T_{0}(\psi)\right|^{2} .
$$

Thus for each $\psi \in \mathcal{K}$ there exists a real valued measurable function $\alpha_{\psi}$ such that

$$
T_{0}(\psi)=e^{i \alpha_{\psi}} T^{3}(\psi) .
$$

Finally (15) and (9) imply that, for any $\phi \in \mathcal{K}$

$$
\left\langle T^{3}(\phi), T^{3}(\phi)\right\rangle=\left\langle T_{0}(\phi), T_{0}(\phi)\right\rangle=\langle\phi, \phi\rangle
$$

and, by polarization, this implies that $T^{3}$ is isometric. Thus $T^{3}$ is a Hilbert algebra endomorphism. Let us denote

$$
T:=T^{3} .
$$

If for some $t>0, \operatorname{supp}(\varphi) \subseteq[-t, t]$ then (15) implies that

$$
\begin{aligned}
e^{\alpha_{\varphi}} T(\varphi) & =T_{0}(\varphi)=T_{0}\left(\varphi \chi_{[-t, t]}\right)=T(\varphi) T_{0}\left(\chi_{[-t, t]}\right) \\
& =e^{i \alpha \chi_{[-t, t]}} T(\varphi) T\left(\chi_{[-t, t]}\right)=e^{i \alpha \chi_{[-t, t]}} T(\varphi) .
\end{aligned}
$$

Thus, for any $\varphi \in \mathcal{K}$ with $\operatorname{supp}(\varphi) \subseteq[-t, t]$, one has, on $\operatorname{supp}(T(\varphi))$ :

$$
\alpha_{\varphi}=\alpha_{\chi_{[-t, t]}} ; \quad \text { a.e. }
$$

In particular, if $s \leq t$

$$
\alpha_{\chi_{[-s, s]}}=\alpha_{\chi_{[-t, t]}} ; \quad \text { a.e. on } \operatorname{supp} T\left(\chi_{[-s, s]}\right) .
$$

Since $T$ is an endomorphism of $\mathcal{K}, T\left(\chi_{[-t, t]}\right)$ is a self-adjoint projection in $\mathcal{K}$, hence it has the form

$$
T\left(\chi_{[-t, t]}\right)=\chi_{I_{t}}
$$


for some measurable subset $I_{t} \subseteq \mathbf{R}$. By the isometry property one can suppose that $t \mapsto I_{t}$ is increasing:

$$
s \leq t \Rightarrow I_{s} \subseteq I_{t} ; \quad \text { a.e. }
$$

Denote

$$
\chi_{I}:=\sup \chi_{I_{t}}=\chi_{\bigcup_{t \geq 0} I_{t}}
$$

since the union can be taken on any sequence increasing to $+\infty$, one can assume that $I$ is measurable up to a set of measure zero. Moreover for any sequence $t_{n} \uparrow+\infty$ the function

$$
\alpha:=\lim \alpha_{\chi_{\left[-t_{n}, t_{n}\right]}}
$$

is well defined on $I$ and measurable up to a sub-set of measure zero of $I$. Because of (16) for any function $\varphi$ with bounded support one has

$$
T_{0}(\varphi)=e^{i \alpha_{\varphi}} T(\varphi)=e^{i \alpha} T(\varphi) .
$$

Since $T_{0}$ is isometric and the functions with bounded support are dense in $\mathcal{K}$, it follows that

$$
T_{0}=e^{i \alpha} T
$$

Conversely, if $T\left(=: T_{3}\right): \mathcal{K} \rightarrow \mathcal{K}$ is a Hilbert algebra endormorphism, $\alpha: \mathbf{R} \rightarrow \mathbf{R}$ a measurable function and $T_{0}, T_{1}, T_{2}$ are defined by (18), (8) respectively, then the map $\tau^{\prime}$, defined by (2) preserves the commutation relations of the RSWN, hence is a $*$-Lie algebra endomorphism.

Finally it is clear that $\tau^{\prime}$ will be an automorphism (i.e. surjective endomorphism) if and only if $T$ is onto, i.e. unitary.

Example 1. A nonsurjective endomorphism of $\mathcal{A}$.

Consider the following isometry on $L^{2}(\mathbf{R})$ :

$$
V f(x)=\left\{\begin{array}{ll}
0 & \text { if } x \in[0,1) \\
f(x-1) & \text { if } x \in[1,+\infty) \\
0 & \text { if } x \in(-1,0] \\
f(x+1) & \text { if } x \in(-\infty,-1]
\end{array} .\right.
$$

One has:

$$
\begin{aligned}
\langle V f, V g\rangle & =\int[V f(x)]^{-}[V g(x)] d x \\
& =\int_{1}^{\infty} \bar{f}(x-1) g(x-1) d x+\int_{-\infty}^{-1} \bar{f}(x+1) g(x+1) d x \\
& =\int_{0}^{\infty} \bar{f}(y) g(y) d y+\int_{-\infty}^{0} \bar{f}(y) g(y) d y=\int_{-\infty}^{+\infty} \bar{f}(y) g(y) d y=\langle f, g\rangle_{L^{2}}
\end{aligned}
$$


Thus $V$ is isometric:

$$
V^{*} V=1
$$

However,

$$
\text { Range } V=\left\{f \in L^{2}(\mathbf{R}): f(x)=0 \text { if } x \in[-1,1]\right\}
$$

which is a proper subspace of $L^{2}(\mathbf{R})$.

Notice that $V$ maps $L^{2} \cap L^{\infty}(\mathbf{R})=: \mathcal{K}$ into itself and clearly induces a Hilbert algebra endomorphism of $\mathcal{K}$. Let us denote it $T^{3}$. Notice however that, if $\chi$ is any characteristic function of a bounded subset in $\mathbf{R}$, then

$$
T^{3}(\chi) \leq \chi_{(-1,1)^{c}} .
$$

Therefore

$$
\bar{\chi}=\sup \left\{T^{(3)} \chi: \chi \text { projectors on bounded subsets of } \mathbf{R}\right\}=\chi_{(-1,1)^{c}}<1 .
$$

Definition 3. The endomorphisms of $\mathcal{A}$, introduced in Theorem 1 are called quasifree. If $\tau^{\prime}$ is such an endomorphism and $(T, \alpha)$ is the pair associated to it through Theorem 1, we shall say that $\tau^{\prime}$ is obtained by SWN quantization (or lifting) of the pair $(T, \alpha)$.

The theorem implies that every quasifree endomorphism $\tau^{\prime}$ of $\mathcal{A}$ can be represented as a composition $\tau^{\prime}=\tau^{1} \tau^{2}$ such that

(A) $\tau^{1}$ is obtained by SWN quantization of the pair $(T, 0)$;

(B) $\tau^{2}$ is obtained by SWN quantization of the pair $(1, \alpha)$.

A quasifree endomorphism will be called of type $(A)$, resp. $(B)$ if it belongs to one of these classes. Notice that, if we consider a one-parameter group of automorphisms with parameter set $\mathbf{R}$, then the associated family of operators $\left(T_{t}\right)_{t \in \mathbf{R}}$ must be a group of endomorphisms in case of type (A) and the function $\alpha_{t}(x)$ must be of the form $\alpha_{t}(x)=\alpha(x) t, t \in \mathbf{R}$, in case of type (B). We are interested to study the spatiality of these evolutions relatively to the representations of $\mathcal{A}$.

Proposition 1. Let $\pi$ be a representation of $\mathcal{A}$ in the Hilbert space $\mathcal{H}=\Gamma\left(\mathcal{H}_{0} \otimes\right.$ $\left.L^{2}(\mathbf{R})\right)$ generated by the Schürmann triple $(\rho, \eta, L)$. Given a type $(\mathbf{A})$ quasifree automorphism $\tau^{\prime}$ of the $S W N$ algebra $\mathcal{A}$, there exists a unitary operator $U$ on $L^{2}(\mathbb{R})$ such that $\tau(\pi(x))=\Gamma(1 \otimes U) \pi(x) \Gamma(1 \otimes U)^{*} ; x \in \mathcal{A}$ where $\Gamma(1 \otimes U)$ denotes the usual second quantization of $U$.

Proof. Let the automorphism $\tau^{\prime}=\tau_{T}^{\prime}$ be obtained by the SWN quantization of the pair $(T, 0)$. To prove the statement we must look for a unitary operator $\mathcal{U}$ on $\mathcal{H}$ implementing $\tau^{\prime}$. By Definition (1) every automorphism of $\mathcal{K}$ can be continued to an automorphism of the von Neumann algebra $L^{\infty}(\mathbf{R})$ that is unitary implementable. Therefore there is a unitary operator $U$ acting on $L^{2}(\mathbf{R})$ such that $T(x)=U x U^{*}$, $x \in \mathcal{K}$. The formula $\mathcal{U} e(f \otimes \phi)=e(f \otimes U \phi), f \in \mathcal{H}_{0}, \phi \in \Gamma\left(L^{2}(\mathbf{R})\right)$, defines a unitary operator $\mathcal{U}=\Gamma(1 \otimes U)$ on the vector space algebraically generated by the 
exponential vectors $e(f \otimes \phi)$ in $\mathcal{H}$ and therefore on the whole of $\mathcal{H}$. The property $\pi\left(\tau^{\prime}(x)\right)=\mathcal{U} \pi(x) \mathcal{U}^{*}, x \in \mathcal{A}$, holds. One can define $\tau(x)=\tau_{T}(x)=\mathcal{U} x \mathcal{U}^{*}, x \in \mathcal{B}(\mathcal{H})$.

Given a type (B) quasifree automorphism $\tau^{\prime}$ of the SWN algebra $\mathcal{A}$ obtained by the lifting of pair $(1, \alpha)$ and a representation $\pi$ of $\mathcal{A}$ generated by the Schürmann triple $(\rho, \eta, L)$, it is natural to conjecture that the associated automorphism $\tau(\cdot)$, of the algebra $\mathcal{B}(\mathcal{H}),\left(\tau(\pi(x))=\pi\left(\tau^{\prime}(x)\right), x \in \mathcal{A}\right)$, has the form $\tau(\cdot)=e^{i H}(\cdot) e^{-i H}$ with

$$
\begin{aligned}
H & =\frac{1}{2} \int \alpha(t) d n_{t}, \\
d n_{t} & =d \Lambda(\rho(M))+d A^{*}(\eta(M))+d A(\eta(M))+(L(M)-\gamma) d t .
\end{aligned}
$$

This conjecture can be proved by an approximation argument which makes use of the following.

Proposition 2. In the above notations let $\alpha$ be a locally constant function vanishing outside a bounded interval and let $\left(\tau_{\varepsilon}\right)$ be the 1-parameter automorphism group of $\mathcal{A}$ associated to the pairs $(1, \varepsilon \alpha), \varepsilon \in \mathbf{R}$. Then for any $x \in \mathcal{A}$

$$
\tau_{\varepsilon}(\pi(x))=e^{i \varepsilon H} \pi(x) e^{-i \varepsilon H} .
$$

Proof. For each $x \in \mathcal{A}$ denote

$$
x(\varepsilon)=\tau_{\varepsilon}(x) .
$$

Choosing $x=b_{\psi}^{+}(\psi \in \mathcal{K})$ one has

$$
\partial_{\varepsilon} \pi\left(b_{\psi}^{+}(\varepsilon)\right)=\partial_{\varepsilon} \pi\left(b_{e^{i \varepsilon \alpha} \psi}^{+}\right)=\pi\left(b_{i \alpha e^{i \varepsilon \alpha} \psi}^{+}\right)=i \alpha \pi\left(b_{\psi}^{+}(\varepsilon)\right),
$$

where in the last identity we have used the fact that, because of the independent increment property, it is sufficient to consider the case $\alpha=$ const. on the support of $\psi$.

In our assumptions the operator $H=H(\alpha)$ is self-adjoint and

$$
C_{\psi}^{+}(\varepsilon):=e^{i \varepsilon H_{\pi}}\left(b_{\psi}^{+}\right) e^{-i \varepsilon H} .
$$

satisfies the equation

$$
\partial_{\varepsilon} C_{\psi}^{+}(\varepsilon)=i e^{i \varepsilon H}\left[H, \pi\left(b_{\psi}^{+}\right)\right] e^{-i \varepsilon H}=i e^{i \varepsilon H} \pi\left(\left[n_{\alpha / 2}, b_{\psi}^{+}\right]\right) e^{-i \varepsilon H} .
$$

It follows that $\tau_{\varepsilon}\left(b_{\psi}^{+}\right)$and $C_{\psi}^{+}(\varepsilon)$ satisfy the same ordinary differential equation with the same initial condition. Since $\alpha$ is constant, in both cases the unique solution is

$$
\tau_{\varepsilon}\left(\pi\left(b_{\psi}^{+}\right)\right)=C_{\psi}^{+}(\varepsilon)=\pi\left(b_{e^{i \varepsilon \alpha} \psi}^{+}\right) .
$$

Similarly one verifies that

$$
\tau_{\varepsilon}\left(\pi\left(b_{\psi}\right)\right)=\pi\left(b_{e^{i \varepsilon \alpha} \psi}\right) ; \quad \tau_{\varepsilon}\left(\pi\left(n_{\psi}\right)\right)=\pi\left(n_{\psi}\right)
$$

and from this (21) follows. 


\section{KMS States Associated with Quasifree Evolutions on the SWN Algebra}

Consider the representations $\rho^{ \pm}$of $\mathcal{U}\left(\mathbf{s l}_{2}\right)$ in $l^{2}$ (cf. Ref. 2) defined by:

$$
\begin{aligned}
& \rho^{+}\left(B^{+}\right) e_{n}=\rho^{-}\left(B^{-}\right) e_{n}=\sqrt{(n+1)(n+2)} e_{n+1}, \\
& \rho^{+}\left(B^{-}\right) e_{n}=\rho^{-}\left(B^{+}\right) e_{n}=\sqrt{n(n+1)} e_{n-1} \\
& \rho^{ \pm}(M) e_{n}= \pm(2 n+2) e_{n},
\end{aligned}
$$

where $\left\{e_{0}, e_{1}, \ldots, e_{n}, \ldots\right\}$ is an orthonormal basis of $\ell^{2}=\ell(\mathbb{N})$. Then define a pair of states $\phi^{ \pm}=\phi_{\lambda}^{ \pm}$on $\mathcal{U}\left(\mathbf{s l}_{2}\right)$ by the formula

$$
\phi_{\lambda}^{ \pm}(\cdot)=(1-\lambda) \sum_{n=0}^{+\infty} \lambda^{n}\left(e_{n}, \rho^{ \pm}(\cdot) e_{n}\right) .
$$

In the representation $\pi=\rho^{+} \otimes \rho^{-}$of $\mathcal{U}\left(\mathbf{s l}_{2}\right) \otimes \mathcal{U}\left(\mathbf{s l}_{2}\right)$ in the Hilbert space $\mathcal{H}_{\phi}=l^{2} \otimes l^{2}$ we get, for any

$$
\begin{array}{r}
\phi_{\lambda}^{+}(x)=\left(\psi_{\lambda}, \pi(x \otimes \mathbf{1}) \psi_{\lambda}\right), \\
\phi_{\lambda}^{-}(x)=\left(\psi_{\lambda}, \pi(\mathbf{1} \otimes x) \psi_{\lambda}\right), \phi_{\lambda}(x \otimes y)=\left(\psi_{\lambda}, \pi(x \otimes y) \psi_{\lambda}\right) \\
x, y \in \mathcal{U}\left(\mathbf{s l}_{2}\right),
\end{array}
$$

where $\psi_{\lambda}=\sqrt{1-\lambda} \sum_{n=0}^{+\infty} \lambda^{\frac{n}{2}} e_{n} \otimes e_{n} \in \mathcal{H}_{\phi}$. So one can consider $\pi$ as the GNS representation associated with the state $\phi_{\lambda}$. Put $B_{1}^{+}=\pi\left(B^{+} \otimes \mathbf{1}\right), B_{1}^{-}=\pi\left(B^{-} \otimes\right.$ 1), $M_{1}=\pi(M \otimes \mathbf{1})$ and $B_{2}^{-}=\pi\left(\mathbf{1} \otimes B^{-}\right), B_{2}^{+}=\pi\left(\mathbf{1} \otimes B^{+}\right), M_{2}=\pi(\mathbf{1} \otimes M)$. Each triple $\left(B_{i}^{+}, B_{i}^{-}, M_{i}\right)(i=1,2)$ satisfies the relations of $\mathbf{s l}_{2}$ by definition.

Proposition 3. The pairwise commuting operators $\left(B_{1}^{+}, B_{1}^{-}, M_{1}\right)$ and $\left(B_{2}^{+}\right.$, $\left.B_{2}^{-}, M_{2}\right)$ satisfy the following relations

$$
\begin{aligned}
& B_{1}^{-} \psi_{\lambda}=\sqrt{\lambda} B_{2}^{-} \psi_{\lambda}, \quad B_{1}^{+} \psi_{\lambda}=\frac{1}{\sqrt{\lambda}} B_{2}^{+} \psi_{\lambda}, \\
& M_{1} \psi_{\lambda}=-M_{2} \psi_{\lambda} .
\end{aligned}
$$

Proof. The identities

$$
\begin{aligned}
B_{1}^{+} e_{n} \otimes e_{n} & =\pi\left(B^{+} \otimes \mathbf{1}\right) e_{n} \otimes e_{n} \\
& =\rho^{+}\left(B^{+}\right) e_{n} \otimes e_{n}=\sqrt{(n+1)(n+2)} e_{n+1} \otimes e_{n} \\
& =e_{n+1} \otimes \rho^{-}\left(B^{+}\right) e_{n+1}=\pi\left(\mathbf{1} \otimes B^{+}\right) e_{n+1} \otimes e_{n+1}=B_{2}^{+} e_{n+1} \otimes e_{n+1}
\end{aligned}
$$

imply

$$
\begin{aligned}
B_{1}^{+} \psi_{\lambda} & =\sqrt{1-\lambda} \sum_{n=0}^{+\infty} \lambda^{\frac{n}{2}} B_{1}^{+} e_{n} \otimes e_{n}=\sqrt{1-\lambda} \sum_{n=1}^{+\infty} \lambda^{\frac{n-1}{2}} B_{2}^{+} e_{n} \otimes e_{n} \\
& =\frac{1}{\sqrt{\lambda}} B_{2}^{+} \psi_{\lambda},
\end{aligned}
$$


where we used the formula $B_{2}^{+} e_{0} \otimes e_{0}=e_{0} \otimes \rho^{-}\left(B^{+}\right) e_{0}=0$. The remaining equalities can be proved in the same way.

Consider the Schürmann triple $\left(\pi, \eta_{\lambda}, \tilde{\phi}_{\lambda}\right)$ consisting of the representation $\pi$ of $\mathcal{U}\left(\mathbf{s l}_{\mathbf{2}}\right) \otimes \mathcal{U}\left(\mathbf{s l}_{2}\right)$ on $\ell^{2} \otimes \ell^{2}$, the trivial cocycle $\eta_{\lambda}(x)=\pi(x) \psi_{\lambda}$ and the conditionally positive functional $\tilde{\psi}_{\lambda}(x)=\left(\psi,(\pi(x)-\varepsilon(x)) \psi_{\lambda}\right), x \in \mathcal{U}\left(\mathbf{s l}_{2}\right) \otimes \mathcal{U}\left(\mathbf{s l}_{2}\right)$, where $\varepsilon$ is the counit on $\mathbf{s l}_{2}$. The restrictions of $\left(\pi, \eta_{\lambda}, \tilde{\phi}_{\lambda}\right)$ give two Schürmann triples $\left(\pi_{ \pm}, \eta_{\lambda, \pm}, \tilde{\phi}_{\lambda, \pm}\right)$ consisting of representations $\pi_{ \pm}$of $\mathcal{U}\left(\mathbf{s l}_{2}\right)$ such that $\pi_{+}(x)=\pi(x \otimes$ $\mathbf{1}), \pi_{-}(x)=\pi(\mathbf{1} \otimes x), \eta_{\lambda, \pm}(x)=\pi_{ \pm}(x) \psi_{\lambda}, x \in \mathcal{U}\left(\mathbf{s l}_{2}\right)$. Then we can define the Levy process $j_{s t}$ over $\mathcal{U}\left(\mathbf{s l}_{2}\right) \otimes \mathcal{U}\left(\mathbf{s l}_{2}\right)$ associated with $\left(\pi, \eta_{\lambda}, \tilde{\phi}_{\lambda}\right)$ by:

$$
\begin{gathered}
j_{s t}(x)=\Lambda_{s t}(\pi(x))+A_{s t}^{*}\left(\eta_{\lambda}(x)\right)+A_{s t}\left(\eta_{\lambda}\left(x^{*}\right)\right)+(t-s) \phi_{\lambda}(x) \mathrm{Id}, \\
x \in \mathcal{U}\left(\mathbf{s l}_{2}\right) \otimes \mathcal{U}\left(\mathbf{s l}_{2}\right),
\end{gathered}
$$

and two Levy processes $j_{s t}^{ \pm}$over $\mathcal{U}\left(\mathbf{s l}_{2}\right)$ associated with $\left(\pi_{+}, \eta_{\lambda,+}, \tilde{\phi}_{\lambda,+}\right)$ and $\left(\pi_{-}, \eta_{\lambda,-}, \tilde{\phi}_{\lambda,-}\right)$ respectively by:

$$
\begin{gathered}
j_{s t}^{+}\left(B^{ \pm}\right)=\Lambda_{s t}\left(B_{1}^{ \pm}\right)+A_{s t}^{*}\left(B_{1}^{ \pm} \psi_{\lambda}\right)+A_{s t}\left(B_{1}^{\mp} \psi_{\lambda}\right)+(t-s)\left(\psi_{\lambda}, B_{1}^{ \pm} \psi_{\lambda}\right) \mathrm{Id}, \\
j_{s t}^{+}(M)=\Lambda_{s t}\left(M_{1}\right)+A_{s t}^{*}\left(M_{1} \psi_{\lambda}\right)+A_{s t}\left(M_{1} \psi_{\lambda}\right)+(t-s)\left(\psi_{\lambda}, M_{1} \psi_{\lambda}\right) \mathrm{Id}, \\
j_{s t}^{-}\left(B^{ \pm}\right)=\Lambda_{s t}\left(B_{2}^{ \pm}\right)+A_{s t}^{*}\left(B_{2}^{ \pm} \psi_{\lambda}\right)+A_{s t}\left(B_{2}^{\mp} \psi_{\lambda}\right)+(t-s)\left(\psi_{\lambda}, B_{2}^{ \pm} \psi_{\lambda}\right) \mathrm{Id}, \\
j_{s t}^{-}(M)=\Lambda_{s t}\left(M_{2}\right)+A_{s t}^{*}\left(M_{2} \psi_{\lambda}\right)+A_{s t}\left(M_{2} \psi_{\lambda}\right)+(t-s)\left(\psi_{\lambda}, M_{2} \psi_{\lambda}\right) \mathrm{Id} .
\end{gathered}
$$

Defining

$$
\begin{aligned}
& \theta_{ \pm}\left(b_{[s, t]}\right):=j_{s t}^{ \pm}\left(B^{-}\right), \quad \theta_{ \pm}\left(b_{[s, t]}^{+}\right):=j_{s t}^{ \pm}\left(B^{+}\right), \\
& \theta_{ \pm}\left(n_{[s, t[}\right):=j_{s t}^{ \pm}(M)-\gamma(t-s) \mathrm{Id} .
\end{aligned}
$$

One obtains two representations $\theta_{ \pm}=\theta_{ \pm}^{(\lambda)}$ of the RSWN algebra $\mathcal{A}$ acting on the same Fock space $\mathcal{H}=\Gamma\left(L^{2}(\mathbb{R}) \otimes \ell^{2} \otimes \ell^{2}\right)=\Gamma\left(\mathcal{H}_{\phi} \otimes L^{2}\right)$ of the tensor product of two SWN algebras $\mathcal{A} \otimes \mathcal{A}$ defined by

$$
\theta(x \otimes y)=\theta_{+}(x) \theta_{-}(y), x, y \in \mathcal{A} .
$$

Proposition 4. The maps $\theta_{ \pm}$define two representations of the RSWN algebra $\mathcal{A}$ in the Hilbert space $\mathcal{H}=\Gamma\left(\mathcal{H}_{\phi} \otimes L^{2}\right)$ such that $\theta_{+}(x)$ and $\theta_{-}(y)$ are commuting for all $x, y \in \mathcal{A}$.

Proof. Notice that $\theta_{+}(x)$ and $\theta_{-}(y)$ commute if $x, y \in \mathcal{A}$ because $B_{1}^{-}, B_{1}^{+}, M_{1}$ and $B_{2}^{-}, B_{2}^{+}, M_{2}$ commute. Hence we need to prove only that $\theta_{+}$and $\theta_{-}$are representations. Using the CCR relations (see the Introduction) we get

$$
\begin{aligned}
{\left[\theta_{+}\left(b_{[s, t[}\right), \theta_{+}\left(b_{[s, t}^{+}\right)\right] } & =\Lambda_{s t}\left(M_{1}\right)+A_{s t}^{*}\left(M_{1} \psi_{\lambda}\right)+A_{s t}\left(M_{1} \psi_{\lambda}\right)+\left(\psi_{\lambda}, M_{1} \psi_{\lambda}\right) \\
& =\theta_{+}\left(n_{[s t}\right)+\gamma(t-s) \mathrm{Id},
\end{aligned}
$$




$$
\begin{aligned}
{\left[\theta_{+}\left(n_{[s, t[}\right), \theta_{+}\left(b_{[s, t]}^{ \pm}\right)\right] } & = \pm \Lambda_{s t}\left(B_{1}^{ \pm}\right) \pm A_{s t}^{*}\left(B_{1}^{ \pm} \psi_{\lambda}\right) \mp A_{s t}\left(B_{1}^{\mp} \psi_{\lambda}\right) \pm\left(\psi_{\lambda}, B_{1}^{ \pm} \psi_{\lambda}\right) \\
& =\theta_{+}\left(b_{[s, t[}^{ \pm}\right) .
\end{aligned}
$$

The remaining formulas can be proved analogously.

Denote $\Omega \in \Gamma\left(L^{2}(\mathbb{R}) \otimes \mathcal{H}_{\phi}\right)$ and define the state $\omega_{\lambda}: \mathcal{A} \rightarrow \mathbf{C}$, by the formula

$$
\omega_{\lambda}(x)=\left(\Omega, \theta_{+}(x) \Omega\right) .
$$

Let $\tau_{\lambda}^{\prime}=\left(\tau_{t}^{\prime}\right)_{t \in \mathbf{R}}$ be the group of type (B) quasifree automorphisms on $\mathcal{A}$ defined by

$$
\begin{gathered}
\tau_{t}^{\prime}\left(b_{\phi}\right)=\lambda^{-i t} b_{\phi}, \quad \tau_{t}^{\prime}\left(b_{\phi}^{+}\right)=\lambda^{i t} b_{\phi}^{+}, \\
\tau_{t}^{\prime}\left(n_{\phi}\right)=n_{\phi}, \quad t \in \mathbf{R}, 0<\lambda<1 .
\end{gathered}
$$

Theorem 2. $\omega_{\lambda}$ is a KMS state on $\mathcal{A}$ associated with the evolution $\tau^{\prime}$, i.e.

$$
\omega_{\lambda}(y x)=\omega_{\lambda}\left(x \tau_{i}^{\prime}(y)\right)
$$

for all elements $x, y \in \mathcal{A}$ which are analytic with respect to $\tau^{\prime}$.

Proof. Notice that $\tau_{i}^{\prime}\left(b_{\chi_{[s, t]}^{ \pm}}^{ \pm}\right)=\lambda^{ \pm 1} b_{\chi_{[s, t[}}^{ \pm}$. Hence it is sufficient to prove only that

$$
\omega_{\lambda}\left(b_{\chi_{[s, t]}^{ \pm}}^{ \pm} y\right)=\lambda^{ \pm 1} \omega\left(y b_{\chi_{[s, t]}^{ \pm}}^{ \pm}\right), y \in \mathcal{A} .
$$

Given $x=\theta_{+}(y) \in \theta_{+}(\mathcal{A})$ one obtains, using Proposition 3

$$
\begin{aligned}
\omega_{\lambda}\left(b_{\chi_{[s, t[}}^{+} y\right)= & \left(\Lambda_{s t}\left(B_{1}^{-}\right) \Omega, x \Omega\right) \\
& +\left(A_{s t}^{*}\left(B_{1}^{-} \psi_{\lambda}\right) \Omega, x \Omega\right)+\left(A_{s t}\left(B_{1}^{+} \psi_{\lambda}\right) \Omega, x \Omega\right) \\
& +(t-s)\left(\psi_{\lambda}, B_{1}^{+} \psi_{\lambda}\right) \omega_{\lambda}(x)=: \mathcal{S} .
\end{aligned}
$$

Notice that

$$
\begin{aligned}
\left(\psi_{\lambda}, B_{2}^{+} \psi_{\lambda}\right) & =\left(\psi_{\lambda}, \mathbf{1} \otimes \rho^{-}\left(B^{+}\right) \psi_{\lambda}\right) \\
& =(1-\lambda)\left(\sum_{n=0}^{+\infty} \lambda^{\frac{n}{2}} e_{n} \otimes e_{n}, \sum_{n=0}^{+\infty} \lambda^{\frac{n}{2}} e_{n} \otimes \sqrt{n(n+1)} e_{n-1}\right)=0 .
\end{aligned}
$$

Hence

$$
0=\left(\psi_{\lambda}, B_{2}^{+} \psi_{\lambda}\right)=\overline{\left(\psi_{\lambda}, B_{2}^{+} \psi_{\lambda}\right)}=\left(B_{2}^{+} \psi_{\lambda}, \psi_{\lambda}\right)=\left(\psi_{\lambda}, B_{2}^{-} \psi_{\lambda}\right) .
$$

Using the last relation, Proposition 4 and the equality $\Lambda_{s t}(x) \Omega=A_{s t}(\xi) \Omega=0, x \in$ $\mathcal{U}\left(\mathbf{s l}_{2}\right), \xi \in \mathcal{H}$, we get the following expression,

$$
\begin{aligned}
\mathcal{S}= & \sqrt{\lambda}\left(\left(A_{s t}^{*}\left(B_{2}^{-} \psi_{\lambda}\right) \Omega, x \Omega\right)+(t-s)\left(\psi_{\lambda}, B_{2}^{+} \psi_{\lambda}\right) \omega_{\lambda}(x)\right) \\
= & \sqrt{\lambda}\left(\left(\Lambda_{s t}\left(B_{2}^{-}\right) \Omega, x \Omega\right)\right. \\
& \left.+\left(A_{s t}^{*}\left(B_{2}^{-} \psi\right) \Omega, x \Omega\right)+\left(A_{s t}\left(B_{2}^{+} \psi\right) \Omega, x \Omega\right)+(t-s)\left(\psi, B_{2}^{-} \psi\right) \omega_{\lambda}(x)\right) \\
= & \sqrt{\lambda}\left(\theta_{-}\left(b_{\chi_{[s t}}\right) \Omega, x \Omega\right)=\sqrt{\lambda}\left(\Omega, x \theta_{-}\left(b_{\chi_{[s t[}^{+}}^{+}\right) \Omega\right)=\lambda \omega_{\lambda}\left(y b_{\chi_{[s, t]}^{+}}^{+}\right) .
\end{aligned}
$$


The equality

$$
\omega_{\lambda}\left(b_{\chi_{[s, t[}} x\right)=\frac{1}{\lambda} \omega_{\lambda}\left(x b_{\chi_{[s, t]}}\right), x \in \mathcal{A}
$$

can be checked in the same way.

In every representation $\theta$ of the algebra $\mathcal{A}$ there are sufficiently many hermitian operators $x \in \theta(\mathcal{A})$. One can apply the functions $f \in L^{\infty}$ to these operators and consider the von Neumann algebra $\mathcal{M}=\theta(\mathcal{A})^{\prime \prime} \wedge \mathcal{B}(\mathcal{H})$ generated by all the $f(x) \in$ $\mathcal{B}(\mathcal{H})$. The von Neumann algebra $\mathcal{M}$ generated by the irreducible representation $\theta$ is of type $I$. Let $\mathcal{M}=\mathcal{M}_{ \pm}$be the von Neumann algebras generated by the representations constructed from the KMS state associated with the evolution $\tau_{\lambda}^{\prime}$ on $\mathcal{A}$. These representations are not irreducible. In particular, the algebras $\mathcal{M}_{+}$ and $\mathcal{M}_{-}$commute.

\section{Acknowledgments}

Grigori Amosov is grateful to Professor Luigi Accardi for kind hospitality during his visit at the Centro Vito Volterra of Università di Roma Tor Vergata.

\section{References}

1. L. Accardi, Y. G. Lu and I. V. Volovich, White noise approach to classical and quantum stochastic calculi, Centro Vito Volterra, Università di Roma "Tor Vergata", preprint 375, 1999.

2. L. Accardi, U. Franz and M. Skeide, Renormalized squares of white noise and other non-Gaussian noises as Levy processes on real Lie algebras, Commun. Math. Phys. 228 (2002) 123-150.

3. U. Farnz, Lévy processes on real Lie algebras, to appear in Proc. of the First SinoGerman Conf. on Stochastic Analysis (A Satellite Conference of ICM 2002), Beijing, China, August 29-September 3, 2002.

4. K. R. Parthasarathy, An Introduction to Quantum Stochastic Calculus (Birkhäuser, 1992).

5. P.-A. Meyer, Quantum Probability for Probabilitists, Lecture Notes in Math., Vol. 1538 (Springer-Verlag, 1995), 2nd edn.

6. A. Connes, Une classification des facteurs de type III, Ann. Sci. Ecole Norm. Sup. 6 (1973) 133-252. 